\title{
FINANCIAL AND STATISTICAL LITERACY FOR RETIREMENT HOUSING DECISIONS IN AUSTRALIA
}

\author{
Timothy Kyng, Ling Li, and Ayse Bilgin \\ Macquarie University Sydney, Australia \\ timothy.kyng@mq.edu.au
}

Many older people in Australia sell their family home to fund a long term residential arrangement with a "retirement village". The contracts are complex. Consumers usually lack the capacity to compare various retirement village contracts with each other or with other arrangements. We have designed a methodology for comparing such contracts via a comparison rent and other metrics. We are working towards developing a free online publicly available calculator and relevant educational material to facilitate informed decision making by consumers. Our proposed calculator will utilise publicly available data on mortality and disability to model survival of resident status. It will compute various metrics that measure the costs, benefits and risks of these contracts. These metrics vary with age, gender, and health characteristics. These freely (soon) available resources are intended to educate both consumers and their advisors / families in statistical, health, and financial literacy when they need to make an important decision towards the end of their lives. 